Transient chronic upgaze differs from that of the transient variety of tonic downgaze deviation of the eyes in newborns, being more persistent and exacerbated by fatigue or illness (see Progress in Pediatric Neurology Millichap ed. 1991, p. 139).

\title{
SHUDDERING ATTACKS
}

The successful treatment of shuddering attacks with a beta-adrenergic blocker (propranolol) in a 3 year old girl is reported from the Division of Pediatric Neurology, Children's Hospital of Philadelphia, University of Pennsylvania, Philadelphia PA. The girl was admitted with a 3 week history of shaking episodes described as jerking and shivering 5 to 6 times a day. There was no loss of consciousness. The problem was first diagnosed as a tic since eye blinking had also been present for several months. The family history was negative for tremor or other movement disorders. The neurological exam showed episodes of head flexion with adduction and flexion of the arms and knees without loss of consciousness or postural tone. Within 2 weeks of starting propranolol $0.5 \mathrm{mg} / \mathrm{kg} / \mathrm{d}$ the movements had ceased. When therapy was discontinued 2 months later, the shuddering resumed within a week and responded once again to propranolol therapy at $1 / 2$ the original dose. A second attempt to discontinue therapy resulted in a prompt return of the shuddering attacks and the necessity for further treatment (Barron TF, Younkin DP. Propranolol therapy for shuddering attacks. Neurology Jan $1992 ; \underline{42}: 258-259)$. (Reprints: Dr. Todd F. Barron, Division of Pediatric Neurology, The Milton S. Hershey Medical Center, P.O. Box 850, Hershey, PA 17033.)

COMMENT. It is proposed that the etiology and response to therapy of both essential tremor and shuddering attacks may be mediated by similar mechanisms. This appears to be the first report of successful treatment of shuddering attacks with propranolol. A family history of essential tremor has previously been reported in patients with shuddering spells and some manifested both shuddering and tremor (Vanasse $M$ et al. Neurology $1976 ; \underline{26}: 1027)$. Shuddering attacks have also been related to an intolerance to monosodium glutamate in children (Reif-Leahrer L, Stemmermann MG. N Engl J Med 1975; 293:1204).

\section{BRAIN TUMORS}

\section{POST-SURGICAL CEREBELLAR MUTISM}

Mutism immediately following removal of a large midline posterior fossa medulluloblastoma and a cerebellar arteriovenous malformation is reported in 2 children from the Department of Neurosurgery, University of Florida, Gainsville, FL. The 7 year old boy with medulluloblastoma showed only a mild dysarthria and truncal ataxia at 3 month follow up and a 15 year old girl with acute posterior fossa bleed and obstructive hydrocephalus was similarly affected by a dysarthria and truncal ataxia when examined at 3 months. A review of the literature disclosed 19 cases of transient mutism after surgical remeval of posterior fossa tumors. The more widespread the 
injury to the midportion of the cerebellum with dentate nuclear involvement, the greater the risk of developing post-operative mutism (Dietze DD Jr., Mickle JP. Cerebellar mutism after posterior fossa surgery. Pediatr Neurosurg; Oct 1991; 16:25-31). (Reprints: Donald D. Dietze, Jr., M.D., Department of Neurosurgery, University of Florida, Box J-265, J. Hillis Miller Health Center, Gainesville, FL 32610.)

COMMENT. The authors advise that this possible complication of posterior fossa surgery should be discussed with patient and parents as part of the preoperative planning. Mutism is not restricted to tumor pathology but may be associated with any acute pathological insult to the midportion of the cerebellum. Fortunately, the mutism resolves completely or to a mild cerebellar dysarthria over a 3 month period. Surgery of midline medulloblastomas is particularly at risk for the development of postoperative mutism.

\section{MANAGEMENT OF INTRACRANIAL NEOPLASMS IN NEUROFIBROMATOSIS}

Recommendations are proposed for the diagnosis and management of CNS neoplasms with NF-1 and NF-2 at the Department of Neurology, Section of Pediatric Neurology, Cleveland Clinic Foundation, Cleveland, Ohio; Phoenix Children's Hospital, Phoenix, AZ; and Children's National Medical Center, George Washington University, Washington, D.C.: (1) MRI is indicated in most childhood patients with NF-1 to screen for visual pathway astrocytomas with substantial visual loss or progressive disease. Treatment should include surgery, irradiation or chemotherapy. (2) Focal areas of signal intensity identified in asymptomatic patients should not be treated as neoplastic unless there is pathologic confirmation. (3) Specific CNS neoplasms (i.e., astrocytomas, ependymomas, meningiomas) should be treated with standard management protocols. (4) Intrinsic brainstem tumors with NF1 should be observed for progressive disease and considered for biopsy and/or resection. (5) Gadolinium-enhanced MRI for acoustic neuromas is indicated in children at risk for NF-2. (6) Formal protocols to clarify the incidence, natural history, and management of intracranial neoplasms in NF-1 and NF-2 should be developed in collaborative national studies (Cohen BH, Kaplan AM, Packer RJ for the Children's Cancer Study Group. Management of intracranial neoplasms in children with neurofibromatosis type 1 and 2 . Pediatr Neurosurg Nov 1991; 16 :66-72). (Reprints: Availability and source not

COMMENT. Optic nerve and tract astrocytomas are the most common intracranial neoplasms in NF-1 with an incidence of 5-15\%. They may present with proptosis, extraocular nerve palsies or nystagmus. Some patients have MRI appearance of "tumor streaking" without mass effect, from the chiasm along the optic tracts and optic radiations. The significance of this streaking is not known. The guidelines for the treatment of visual pathway tumors is controversial, especially for asymptomatic patients or patients with non-progressive defects. For an overview of CNS tumors and neurofibromatosis see Progress in Pediatric Neurology, Millichap ed. 1991, pp. 374-379. 